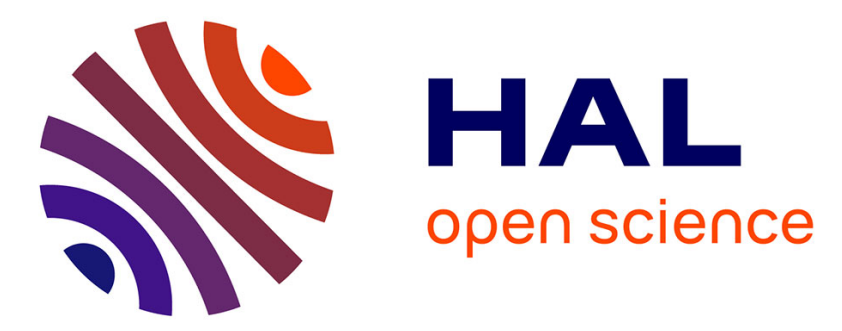

\title{
Influence de l'apport de composés organiques sur l'adsorption et la désorption des ions phosphates en sol ferrallitique
}

\author{
Emmanuel Frossard, Binh Truong, Fernand Jacquin
}

\section{- To cite this version:}

Emmanuel Frossard, Binh Truong, Fernand Jacquin. Influence de l'apport de composés organiques sur l'adsorption et la désorption des ions phosphates en sol ferrallitique. Agronomie, 1986, 6 (6), pp.503-508. 10.1051/agro:19860601 . hal-00884903

\section{HAL Id: hal-00884903 https://hal.science/hal-00884903}

Submitted on 1 Jan 1986

HAL is a multi-disciplinary open access archive for the deposit and dissemination of scientific research documents, whether they are published or not. The documents may come from teaching and research institutions in France or abroad, or from public or private research centers.
L'archive ouverte pluridisciplinaire $\mathbf{H A L}$, est destinée au dépôt et à la diffusion de documents scientifiques de niveau recherche, publiés ou non, émanant des établissements d'enseignement et de recherche français ou étrangers, des laboratoires publics ou privés. 


\title{
Influence de l'apport de composés organiques sur l'adsorption et la désorption des ions phos- phates en sol ferrallitique
}

\author{
Emmanuel FROSSARD, Binh TRUONG (*) \& Fernand JACQUIN \\ E.N.S.A.I.A., Département de Sciences du Milieu, 2, avenue de la Forêt-de-Haye, F 54500 Vandoeuvre \\ (*) I.R.A.T./C.I.R.A.D., Laboratoire de Radioagronomie, avenue du Val-de-Montferrand, B.P. 5035, \\ F 34032 Montpellier Cedex
}

\begin{abstract}
Nous avons étudié l'influence de deux composés organiques (glucose, xanthane) sur l'adsorption et la désorption des ions phosphates dans deux horizons (A et B) d'un sol ferrallitique forestier. Cette recherche a été menée après une incubation de 21 jours sous paramètres contrôlés. Les substrats organiques n'ont eu que peu d'effets sur le comportement de l'horizon supérieur ; les matières organiques préexistantes occuperaient déjà la plupart des sites de fixation de ce sol. Par contre, dans l'horizon profond, les apports de glucose et de xanthane ont nettement diminué l'adsorption et augmenté la désorption des ions phosphates. Ils auraient permis la saturation relative des sites d'adsorption et entraîné la création de composés phosphorés d'où les ions phosphates seraient facilement désorbés. Dans les sols ferrallitiques cultivés, appauvris en matières organiques, des apports combinés d'engrais phosphatés et d'amendements organiques fermentescibles pourraient donc améliorer l'assimilabilité des ions phosphates et le coefficient d'utilisation du $\mathbf{P}$ des engrais.
\end{abstract}

Mots clés additionnels : Phosphore isotopiquement diluable, matière organique, engrais phosphatés.

\begin{abstract}
Influence of organic matter on adsorption and desorption of phosphorus in an oxisol
We studied the influence of two organic compounds (glucose and xanthan) on adsorption and desorption of phosphorus by two horizons (A and B) of an oxisol. The measurements were made after a 21 -day incubation period, under controlled conditions. The organic compounds added had only little effect on the upper horizon, since existing organic matter must occupy almost all fixation sites. In the B horizon, however, glucose and xanthan treatments decreased phosphorus adsorption and increased desorption, presumably by coating the sites and creating phosphate compounds from which phosphorus could be easily desorbed. So we can guess that applying phosphorus fertilizers and manure together in cultivated oxisols, poor in organic matter, will increase the availability of phosphorus and the utilization coefficient of the phosphate fertilizer.
\end{abstract}

Additional key words : Isotopically dilutable phosphorus, organic matter, phosphorus fertilizers.

\section{INTRODUCTION}

L'importance des déficiences en phosphore dans les sols ferrallitiques est bien connue ainsi que la difficulté de les surmonter par des apports même élevés d'engrais phosphatés (FARDEAU \& JAPPE, 1980; FOX, 1980). Elle est liée à la richesse de ces sols en oxyhydroxydes qui présentent de forts pouvoirs fixateurs vis-à-vis des ions phosphates (PARFITT, 1978). Or certains anions organiques possèdent les mêmes potentialités de fixation sur ces minéraux (STUMM et al., 1980); ces composés organiques seraient donc susceptibles de modifier la fixation des ions phosphates dans les sols.

Ainsi, FARDEAU et al. (1977) notent que la diminution de la teneur en carbone d'un sol de grande culture (champ DEHÉRAIN, à Grignon) augmente son pouvoir fixateur vis-à-vis des ions phosphates, puis FARDEAU \& JAPPE (1980) montrent que l'apport de fumier diminue le pouvoir fixateur d'un sol dégradé de Casamance et augmente la teneur en ions phosphates dans la solution du sol.

Il est donc important de rappeler quels sont les mécanismes de fixation (terme général) des ions phos- 
phates dans les sols. Ils peuvent s'adsorber soit par des réactions d'échange de ligand sur les sites $\mathrm{MOH}$ et $\mathrm{MOH}_{2}^{+}$de la kaolinite et des oxyhydroxydes, soit par des liaisons électrostatiques. Ils peuvent aussi être inclus (pénétration des ions entre les feuillets d'argiles) ou occlus (substitution anionique dans une particule minérale).

Nous proposons d'analyser l'influence de l'apport de composés organiques chimiquement définis (glucose, xanthane) sur la capacité d'adsorption pour les ions phosphates de 2 échantillons de sol ferrallitiques et sur les possibilités de désorption de ces ions.

\section{MATÉRIELS ET MÉTHODES}

\section{A. Matériels}

Le sol utilisé est un sol ferrallitique désaturé en provenance du Brésil. Les principales caractéristiques des horizons $\mathrm{A}$ et $\mathrm{B}$ sont décrites dans le tableau 1. Il nous faut noter que ce sol a été prélevé sous couvert forestier, ce qui explique la forte teneur en matière organique de l'horizon supérieur.

Le xanthane est un exopolysaccharide produit par des bactéries de l'espèce Xanthomonas campestris. Ce composé, difficilement dégradable (SANDFORD et al., 1977), possède des groupements carboxyliques (LAWSON \& SYMES, 1977) susceptibles de réagir avec les sites $\mathrm{MOH}$ et $\mathrm{MOH}_{2}^{+}$(où $\mathrm{M}=\mathrm{Al}$ ou Fe) de certains constituants des sols. Le glucose correspond à un substrat très énergétique dont la biodégradation engendre d'importantes quantités de composés organiques susceptibles de s'adsorber sur le sol (GUCKERT, 1973).

\section{B. Méthodes}

\section{Technique d'incubation}

Des échantillons de $100 \mathrm{~g}$ de sol sec tamisé à $2 \mathrm{~mm}$, humidifiés à 80 p. 100 de la capacité de rétention sont incubés pendant $21 \mathrm{j}$ à $28{ }^{\circ} \mathrm{C}$ selon un protocole décrit précédemment (JACQUIN et al., 1979). Les substrats organiques sont apportés à 2 doses correspondant à 5 et à 20 p. 100 du carbone organique total préexistant dans l'horizon A (C.O.T.A ). Le glucose a été apporté en solution ; par contre, le xanthane non soluble a été mélangé au sol avant son humectation.

\section{Adsorption et désorption des ions $\mathrm{PO}_{-4}^{3^{-}}$}

\section{a) Adsorption}

Nous avons utilisé la méthode décrite par TRUONG et al. (1974). Cependant, étant donné les fortes variations de $\mathrm{pH}$ observées entre les traitements à l'issue de l'incubation, nous avons dû ajuster le $\mathrm{pH}$ à 4,1 pour l'horizon $\mathrm{B}$ et à 5,0 pour l'horizon supérieur (valeurs correspondant aux $\mathrm{pH}$ observés dans les sols témoins) ; aussi, différents volumes de $\mathrm{NaOH}$ ou de $\mathrm{HCl} 0,01 \mathrm{M}$ et $7,5 \mathrm{ml}$ de $\mathrm{KCl} 0,02 \mathrm{M}$ sont apportés à $3 \mathrm{~g}$ de sol, ainsi que de l'eau déminéralisée afin d'obtenir un volume de $15 \mathrm{ml}$ et une concentration finale de $0,01 \mathrm{M}$ en $\mathrm{KCl}$. La valeur d'équilibre du $\mathrm{pH}$ s'obtient après $4 \mathrm{j}$ avec 2 agitations journalières. A ces suspensions sont ensuite ajoutées des solutions de $15 \mathrm{ml}$ de $\mathrm{KCl} \mathrm{0,01} \mathrm{M}$ contenant des doses croissantes de phosphore sous la forme $\mathrm{KH}_{2} \mathrm{PO}_{4}$. Celles-ci varient de 20 à $1000 \mu \mathrm{g} \mathrm{P} / \mathrm{g}$ de sol et les solutions sont ajus-

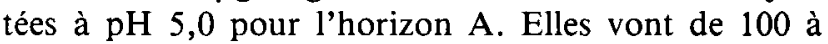
$2000 \mu \mathrm{g} \mathrm{P} / \mathrm{g}$ de sol et sont ajustées à $\mathrm{pH} \mathrm{4,1} \mathrm{pour}$ l'horizon B. Après $20 \mathrm{~h}$ d'agitation, ces suspensions sont centrifugées à $10000 \mathrm{~g}$ pendant $5 \mathrm{mn}$ et décantées. Les tubes sont pesés avant et après décantation afin de déterminer le volume de solution restant dans l'échantillon. La concentration en phosphore de la solution est dosée par colorimétrie du complexe phosphomolybdique en milieu sulfurique. La quantité de phosphore adsorbée est calculée à partir des concentrations initiale et finale de la solution. L'essai est conduit avec 2 répétitions.

Nous utilisons l'équation de l'isotherme de LANGMUIR (OLSEN \& WANATABE, 1957) afin de comparer l'adsorption du phosphore entre les 2 horizons et leurs différents traitements.

$$
\frac{\mathrm{C}}{\mathrm{P}}=\frac{1}{\mathrm{~b}} \mathrm{C}+\frac{1}{\mathrm{bK}}
$$

$\mathrm{C}$ : concentration en phosphore de la solution d'équilibre, exprimée en ppm,

$\mathrm{P}$ : phosphore adsorbé sur le sol, exprimé en ppm par rapport au sol,

b : capacité maximale d'adsorption exprimée en ppm par rapport au sol

$\mathrm{K}$ : coefficient relatif à l'énergie d'adsorption des ions phosphates sur le sol.

TABLEAU

Caractéristiques principales du sol ferrallitique.

Main characteristics of the oxisol.

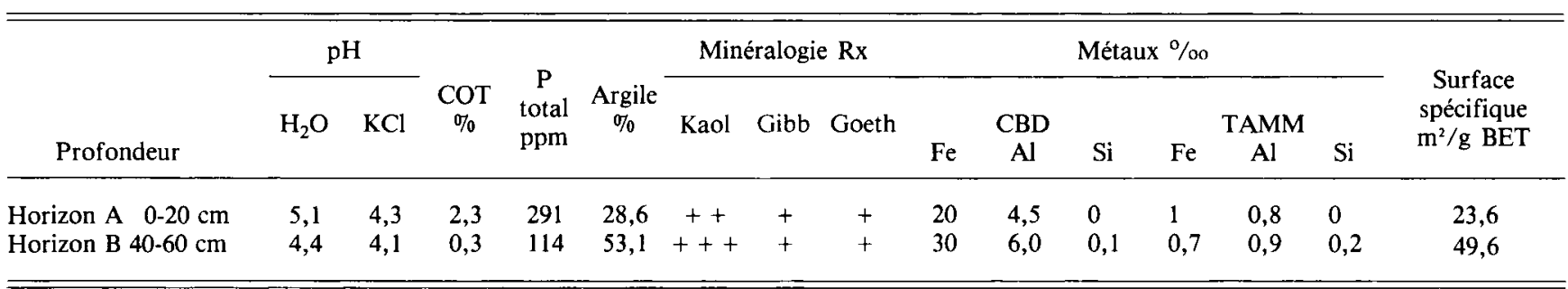

COT : carbone organique total ; RX : rayon X ; Kaol : kaolinite ; Gibb : gibbsite ; Goeth : goethite ; +++ : très abondant ; ++ : abondant ; + : présent ; CBD : citrate, bicarbonate, dithionite. 


\section{b) Désorption}

On agite pendant $4 \mathrm{~h}$ le sol resté dans le tube avec $30 \mathrm{ml}$ de $\mathrm{KCl} 0,01 \mathrm{M}$ contenant du ${ }^{32} \mathrm{P}$ sous la forme $\mathrm{H}_{3}^{32} \mathrm{PO}_{4}$ sans entraîneur. La solution est centrifugée puis décantée. Le dosage du phosphore en solution permet de connaître le phosphore désorbé, en retranchant la quantité provenant de la solution d'équilibre restée dans l'échantillon après adsorption. La mesure de la radioactivité permet de connaître le phosphore isotopiquement diluable.

$\mathrm{P}$ isotopiquement diluable $=$

$$
={ }^{32} \mathrm{P} \text { introduit } \times \frac{{ }^{31} \mathrm{P} \text { désorbé }}{{ }^{32} \mathrm{P} \text { final en solution }}
$$

\section{RÉSULTATS ET DISCUSSION}

\section{A. Comparaison des sols témoins}

L'examen des figures 1 et 2 montre que les courbes d'adsorption des 2 échantillons témoins ont une même allure correspondant au type $« \mathrm{H} »$ mentionné par

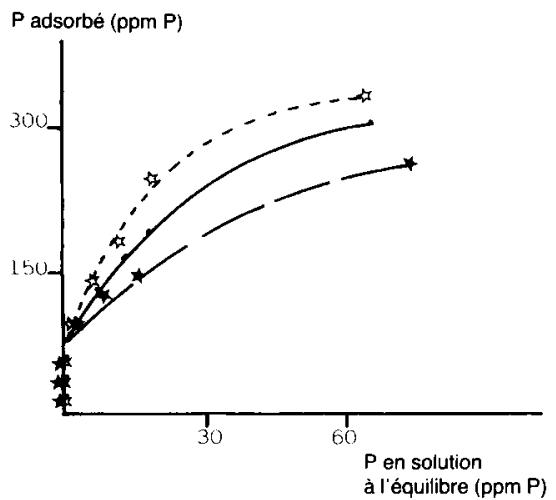

Figure 1

Courbes d'équilibres entre les ions phosphates adsorbés par l'horizon $A$ et les ions phosphates en solution.

Equilibrium curves between phosphorus adsorbed by the $A$ horizon and phosphorus in solution.

- - Sol témoin

$\star--\star \mathrm{Sol}+20 \% \mathrm{COT}_{A}$ xanthane

约..... 5 Sol $+20 \% \mathrm{COT}_{A}$ glucose

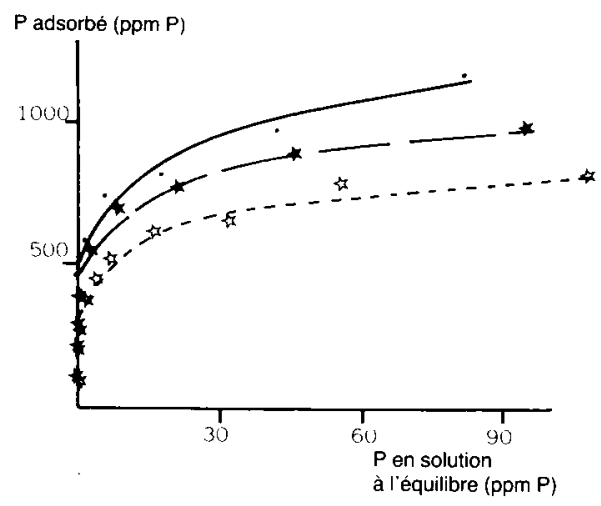

Figure 2

Courbes d'équilibres entre les ions phosphates adsorbés par l'horizon $B$ et les ions phosphates en solution.

Equilibrium curves between phosphorus adsorbed by the $B$ horizon and phosphorus in solution.

- Sol témoin

$\star--\star \mathrm{Sol}+20 \% \mathrm{COT}_{A}$ xanthane

计..... $\mathrm{Sol}+20 \% \mathrm{COT}_{A}$ glucose

GILES et al. (1960), indiquant une forte affinité des ions phosphates pour les sols et qu'elles sont décrites correctement par l'équation de LANGMUIR (tabl. 2). Si ces courbes ont même allure, on note toutefois que l'horizon A du sol brésilien adsorbe beaucoup moins d'ions phosphates que l'horizon profond; ceci doit être relié à la surface spécifique beaucoup plus élevée de ce dernier.

Les valeurs expérimentales (tabl. 2) obtenues lors de la désorption sont beaucoup plus élevées ( $P$ désorbé et $P$ isotopiquement diluable) dans l'horizon A que dans l'horizon profond. La fixation très réversible des ions phosphates dans l'horizon de surface doit être liée à son taux élevé de matières organiques préexistantes.

\section{B. Action des traitements sur l'adsorption des ions phosphates}

\section{Horizon $A$ du sol ferrallitique}

Les courbes d'adsorption (fig. 1) comme les paramètres de l'équation de LANGMUIR (tabl. 3) indiquent

TABLEAU 2

Adsorption et désorption du phosphore dans les horizons $A$ et $B$ sans apports de composés organiques. Adsorption and desorption of phosphorus in the $A$ and $B$ horizons without organic matter.

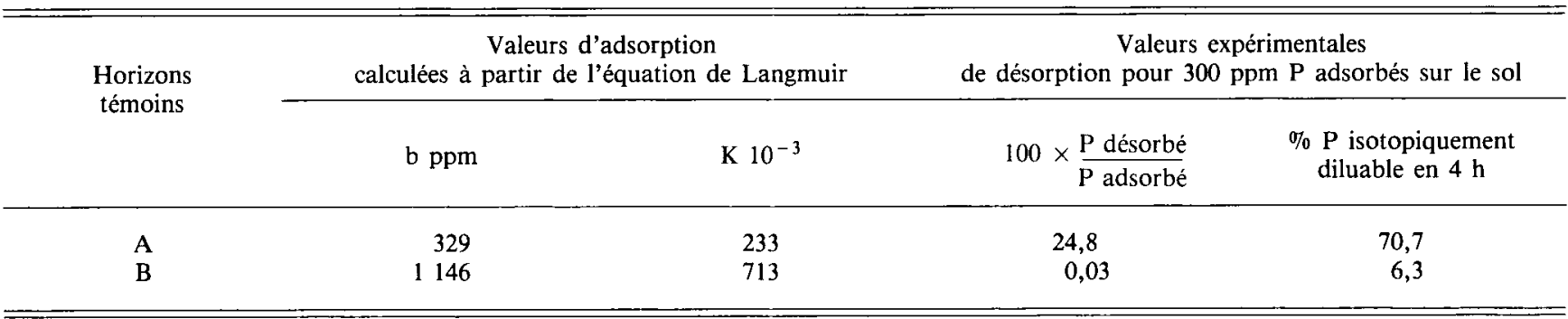


TABLEAU 3

Evolution du $p H$ et des paramètres $b$ et $K$ de l'équation de LANGMUIR dans les horizons $A$ et $B$ d'un sol ferrallitique soumis à des apports de composés organiques.

$p H$ and $b$ and $K$ parameters of the LANGMUIR equation in the $A$ and $B$ horizons of an oxisol with added organic matter.

\begin{tabular}{|c|c|c|c|c|c|c|c|c|c|c|}
\hline & \multicolumn{5}{|c|}{ Horizon A } & \multicolumn{5}{|c|}{ Horizon B } \\
\hline & \multirow[b]{2}{*}{ Témoin } & \multicolumn{2}{|c|}{ glucose } & \multicolumn{2}{|c|}{ xanthane } & \multirow[b]{2}{*}{ Témoin } & \multicolumn{2}{|c|}{ glucose } & \multicolumn{2}{|c|}{ xanthane } \\
\hline & & $5 \% \mathrm{COT}_{\mathrm{A}}$ & $20 \% \mathrm{COT}_{\mathrm{A}}$ & $5 \% \mathrm{COT}_{\mathrm{A}}$ & $20 \% \mathrm{COT}_{\mathrm{A}}$ & & $5 \% \mathrm{COT}_{\mathrm{A}}$ & $20 \% \mathrm{COT}_{\mathrm{A}}$ & $5 \% \mathrm{COT}_{\mathrm{A}}$ & $20 \% \mathrm{COT}_{\mathrm{A}}$ \\
\hline b ppm $P$ & 329 & 258 & 329 & 306 & 270 & 1146 & 963 & 839 & 1042 & 992 \\
\hline $\mathrm{K}^{1} 0^{-3}$ & 233 & 519 & 292 & 258 & 270 & 713 & 366 & 347 & 758 & 509 \\
\hline $\mathrm{pH}$ fixation & 5,0 & 4,9 & 4,9 & 5,0 & 4,9 & 4,3 & 4,0 & 4,2 & 4,2 & 3,9 \\
\hline
\end{tabular}

que, pratiquement, tous les apports de composés organiques abaissent sensiblement la capacité maximale d'adsorption (b), sauf le traitement glucose à 20 p. 100. Cependant, ces différences ne sont nettes que pour des valeurs de $\mathrm{P}$ apportées supérieures à $200 \mathrm{ppm}$, les concentrations de $\mathrm{P}$ obtenues pour des apports plus faibles sont voisines pour tous les traitements. Il semble donc que la fixation du xanthane et des produits de dégradation du glucose sur le sol n'ont eu que peu d'influence sur le devenir des ions phosphates ajoutés.

\section{Horizon $B d u$ sol ferrallitique}

Dans cet horizon, tous les traitements provoquent des chutes très nettes de l'adsorption des ions phosphates (fig. 2) qui se traduisent par des diminutions de la valeur $b$ et de la constante $K$, à l'exception du traitement xanthane à $5 \mathrm{p} .100$ pour cette dernière (tabl. 3). Ces diminutions sont plus fortes lorsque le composé organique est apporté à haute dose, les produits de dégradation du glucose apparaissant plus efficaces que le xanthane à diminuer l'adsorption des ions phosphates. Il est possible d'émettre l'hypothèse suivante : le xanthane, le glucose et/ou ses produits de dégradation se sont fixés sur des sites initialement disponibles pour les ions phosphates.

\section{Action des traitements sur la désorption des ions phosphates}

\section{Horizon $A$ du sol ferrallitique}

Dans cet échantillon, les apports de composés organiques n'ont que peu d'effet sur les pourcentages de phosphore désorbé et de phosphore isotopiquement diluable en $4 \mathrm{~h}$; rappelons que ces pourcentages sont déjà très élevés dans le sol témoin (fig. 3 et 4 ). Ceci permet de dire que les matières organiques préexistantes satureraient pratiquement tous les sites de fixation existant dans ce sol, ne laissant aux ions phosphates que des sites (dont l'énergie de liaison avec les ions $\mathrm{PO}_{4}$ est faible) permettant une désorption facile des ions adsorbés.

\section{Horizon $B$ du sol ferrallitique}

Dans l'échantillon prélevé en profondeur, les apports de composés organiques ont nettement augmenté les pourcentages de ions phosphates désorbés et isotopiquement diluables (fig. 5 et 6). Le substrat le plus effi-

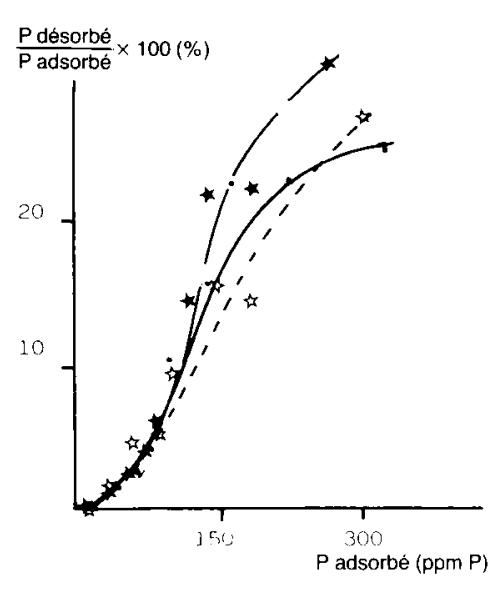

Figure 3

Désorption des ions phosphates de l'horizon $\mathrm{A}$ par $\mathrm{KCl} 0,01 \mathrm{M}$. Phosphorus desorption from A horizon with $0.01 \mathrm{M} \mathrm{KCl}$.

- - Sol témoin

$\star--\star$ Sol $+20 \% \mathrm{COT}_{A}$ xanthane

动-..- $\mathrm{Sol}+20 \% \mathrm{COT}_{A}$ glucose

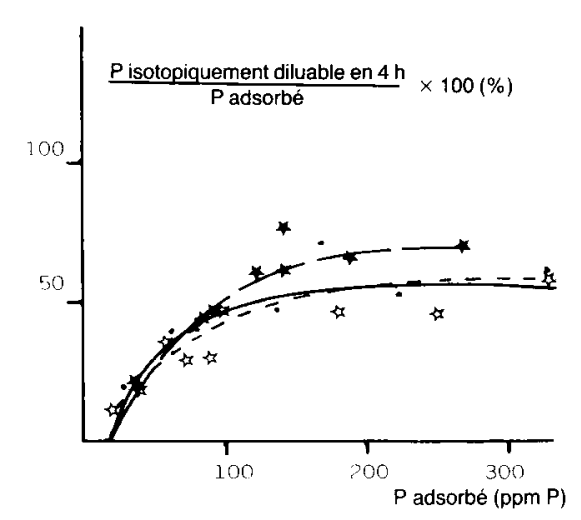

Figure 4

Phosphore isotopiquement diluable de l'horizon A dans $\mathrm{KCl} 0,01 \mathrm{M}$ en $4 \mathrm{~h}$. Phosphorus isotopically dilutable from $A$ horizon within $4 \mathrm{~h}$ in $0.01 \mathrm{M} \mathrm{KCl}$.

•-_... Sol témoin

$\star--\star$ Sol $+20 \% \mathrm{COT}_{A}$ xanthane

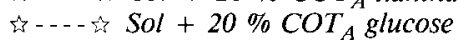

cace est le glucose, suivi du xanthane, comme pour l'adsorption. Ainsi lorsque $500 \mathrm{ppm}$ de phosphore sont adsorbés par le sol, $150 \mathrm{ppm}(30 \mathrm{p} .100)$ sont isotopiquement diluables dans le sol témoin, contre $210 \mathrm{ppm}$ 


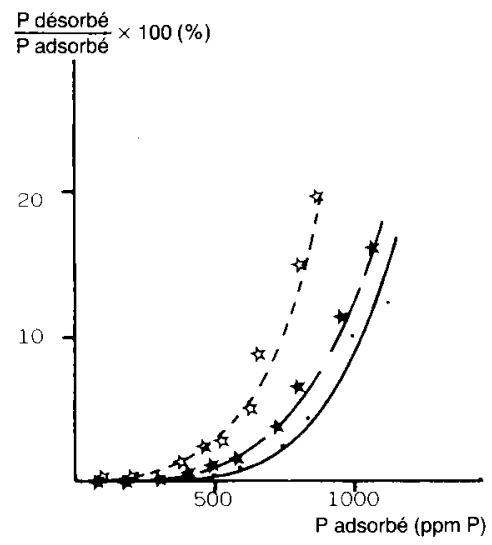

Figure 5

Désorption des ions phosphates de l'horizon $\mathrm{B}$ par $\mathrm{KCl}$ 0,01 $\mathrm{M}$. Phosphorus desorption with $0.01 \mathrm{M} \mathrm{KCl}$ from $B$ horizon.

- - Sol témoin

$\star--\star \mathrm{Sol}+20 \% \mathrm{COT}_{A}$ xanthane

औ-...宛 Sol $+20 \% \mathrm{COT}_{A}$ glucose

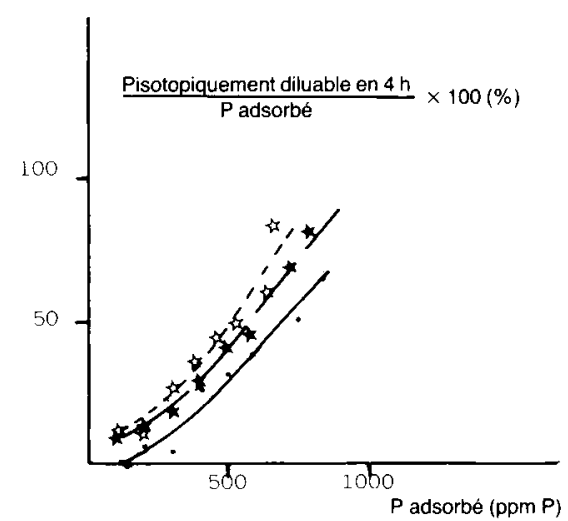

Figure 6

Phosphore isotopiquement diluable de l'horizon $\mathrm{B}$ dans $\mathrm{KCl}$ 0,01 M en $4 \mathrm{~h}$.

Phosphorus isotopically dilutable from $B$ horizon within $4 \mathrm{~h}$ in $0.01 \mathrm{M} \mathrm{KCl}$.

\section{-. Sol témoin}

$\star--$ Sol $+20 \% \mathrm{COT}_{A}$ xanthane

A...- th Sol $+20 \% \mathrm{COT}_{A}$ glucose

(42 p. 100) dans le sol +20 p. $100 \mathrm{COT}_{\mathrm{A}}$ xanthane et 250 ppm (50 p. 100) dans le traitement sol +20 p. 100 $\mathrm{COT}_{\mathrm{A}}$ glucose. Ces faits pourraient être expliqués en supposant que les apports de xanthane et de glucose favoriseraient la création de complexes phosphorés d'où les ions phosphates seraient facilement désorbés.

\section{CONCLUSION}

A l'issue de cette étude, il est possible de proposer quelques mécanismes permettant au xanthane et aux produits de dégradation du glucose d'influencer, dans nos conditions expérimentales, l'adsorption d'ions phosphates en sols ferrallitiques.

Dans l'horizon supérieur du sol ferrallitique considéré, l'apport des différents substrats n'entraîne pas de variations nettes de la capacité d'adsorption et des possibilités de désorption.

Dans ce sol, les sites de fixation des oxyhydroxydes et de la kaolinite seraient saturés, pour la plupart, par les matières organiques initiales ; l'adsorption des ions phosphates aurait lieu sur des sites de plus faible énergie permettant une forte désorption.

Le xanthane et, plus encore, les produits de dégradation du glucose diminuent l'adsorption des ions phosphates dans l'horizon profond et augmentent leur désorption. Ces composés organiques, d'une part, se seraient donc fixés sur les sites initialement disponibles pour les ions phosphates de la kaolinite et des oxyhydroxydes et, d'autre part, auraient favorisé la création de composés phosphorés d'où les ions phosphates seraient facilement désorbés.

Enfïn, bien que ces données ne soient évidemment pas extrapolables au champ, il est possible d'émettre des hypothèses quant à l'efficacité de l'apport de composés organiques fermentescibles sur la fertilisation phosphorique des sols tropicaux. Ces apports ne devraient avoir d'effets nets que sur les sols pauvres en matières organiques préexistantes. Or la mise en culture de sols ferrallitiques forestiers provoque une rapide diminution de leurs teneurs en matières organiques et plus particulièrement les polysaccharides (GODEFROY \& JACQUIN, 1975). Il serait intéressant, dans ces sols, de coupler les apports d'engrais phosphatés avec des amendements organiques fermentescibles (boues résiduaires de stations d'épuration) et résidus de récoltes qui favoriseraient l'apparition de composés transitoires ; ces apports combinés permettraient une meilleure assimilabilité des ions phosphates, augmentant ainsi le coefficient d'utilisation des engrais phosphatés souvent très faible dans ces sols.

Reçu le 23 mai 1985. Accepté le 31 janvier 1986.

\section{REMERCIEMENTS}

Les auteurs remercient l'Institut Mondial des Phosphates pour sa participation financière.

\section{RÉFÉRENCES BIBLIOGRAPHIQUES}

Fardeau J. C., Jappe J., 1980. Choix de la fertilisation phosphorique des sols tropicaux: emploi du phosphore 32. Agron. trop., XXX (3), 225-231.

Fardeau J. C., Chabouis C., Hétier J. M., 1977. Méthode de cinétiques de dilution isotopique des ions $\mathrm{PO}_{4}$ et $\mathrm{K}$ : application à l'analyse des modifications de fertilité dans les essais de très longue durée. Sci. Sol, (2), 111-122.
Fox R. L., 1980. Soils with variable charge : agronomic and fertility aspects, 195-224. In: B. K. G. Theng: "Soils with variable charge ». N.Z. Soc. Soil Sci., 448 p.

Giles C. H., McEwan T. H., Nakhawa S. N., Smith D., 1960. Studies in adsorption. XI. A system of classification of solution adsorption isotherm, and its uses in diagnosis of adsorption mechanisms and in measurement of specific surface area of solids. $D . J$. Chem. Soc., 3972-3993. 
Godefroy J., Jacquin F., 1975. Relation entre la stabilité structurale des sols cultivés et les apports organiques en conditions tropicales ; comparaison avec les sols forestiers. Fruits, 30 (10), 595-612.

Guckert A., 1973. Contribution à l'étude des polysaccharides dans les sols et leur rôle dans les mécanismes d'agrégation. Thèse Doct. Etat, Univ. Nancy I, 124 p.

Jacquin F., Mallouhi N., Gallali T., 1979. Etude sur l'intensité des transferts de matières organiques sous l'influence de la salinité. C. R. Acad. Sci. Paris, 289, D, 1229-1232.

Lawson C. J., Symes K. C., 1977. Xanthan gum, acetolysis as a tool for elucidation of structure, 183-191. In P. A. Sandford \& A. Laskin : "Extracellular microbial polysaccharides ». Washington, D.C., Am. Chem. Soc.

Olsen S. R., Wanatabe F. S., 1957. A method to determine a phosphorus adsorption maximum of soils as measured by the LANGMUIR isotherm. Soil Sci. Soc. Am. Proc., 21, 144-149.
Parfitt R. L., 1978. Anion adsorption by soil and soil material. Adv. Agron., 30, 1-50.

Sandford P. A., Pittsley J. E., Knuston C. A., Watson P. R., Cadmus M. C., Jeanes A., 1977. Variations in Xanthomonas campestris NRRL B-1459 : characterization of xanthan products of differing pyruvic acid content, 192-210. In P. A. Sandford \& A. Laskin : «Extracellular microbial polysaccharides ». Washington, D.C. Am. Chem. Soc.

Stumm X., Kummert R., Sigg L., 1980. A ligand exchange model for the adsorption of inorganic and organic ligands at hydrous oxyde interfaces. Croat. Chem. Acta., 53 (2), 291-312.

Truong B., Burdin S., Bertrand R., Pichot J., 1974. Contribution à l'étude du phosphore dans les sols dérivés de roches volcaniques de l'île de la Réunion (Mascareignes). Actions du carbonate et du silicate de calcium. Agron. trop., XXIX (6-7), 1-12. 